\title{
Pandemia y percepciones docentes acerca de la enseñanza remota de emergencia: El caso de Uruguay
}

\author{
Pandemic and Teacher's Perceptions About Emergency Remote Teaching: The Uruguayan Case
}

\author{
Pandemia e percepção dos professores sobre educação remota de emergência: \\ o caso do Uruguai
}

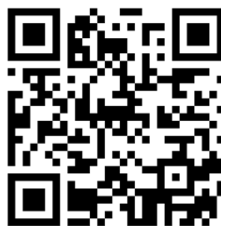

Resumen:

Introducción. Este artículo presenta resultados para el caso Uruguay de una investigación internacional que indagó en la percepción del personal docente acerca de la enseñanza remota de emergencia, en un contexto nacional con dos características distintivas: acceso universal a recursos tecnológicos para la enseñanza por la cobertura nacional del Plan Ceibal y continuidad educativa en línea. Objetivo: La finalidad del estudio fue conocer los cambios generados en los procesos de enseñanza, debido a la emergencia sanitaria provocada por la COVID-19 e indagar sobre los principales retos pedagógicos percibidos por el personal docente. Metodología. El estudio se basó en un diseño descriptivo mixto que integró datos cuantitativos y cualitativos. La información fue recolectada por medio de una encuesta digital autoadministrada aplicada entre mayo y junio de 2020 a 470 docentes que se desempeñan en diferentes niveles de enseñanza, desde educación inicial hasta la universidad. Resultados. Aún en un país como Uruguay con alta conectividad educativa, la suspensión de clases presenciales por

Recibido • Received • Recebido: 27 / 09 / 2020

Corregido • Revised • Revisado: 25 / 09 / 2021

Aceptado • Accepted • Aprovado: 11 / 12 / 2021 Montevideo, Uruguay vaillant@ort.edu.uy

Eduardo Rodríguez-Zidán

Universidad ORT Uruguay

Montevideo, Uruguay

cerzidan@yahoo.com.ar

Mariela Questa-Torterolo

Universidad ORT Uruguay

Montevideo, Uruguay

questa@ort.edu.uy

https://orcid.org/0000-0002-4321-2340 
https://doi.org/10.15359/ree.26-1.4

https://www.revistas.una.ac.cr/index.php/educare

educare@una.ac.cr

la COVID-19 provocó tensiones y desafíos en el plano laboral, pedagógico y tecnológico. Existió un impacto negativo en la salud ocupacional y el estrés docente. Además, se registraron problemas de baja apropiación y uso pedagógico de las tecnologías por parte del personal docente. Discusión. Uno de los grandes retos señalados por el personal docente refiere a la construcción de nuevos formatos pedagógicos para la mejora de los resultados educativos con equidad.

Palabras claves: Aprendizaje en línea; práctica pedagógica; pandemia; docencia.

\begin{abstract}
:
Introduction. This article presents the results of an international research study for the Uruguayan case. The research analyzed teachers' perceptions of emergency remote teaching in a national context. This context had two distinctive characteristics: universal access to technological resources for teaching due to the national coverage of the Ceibal Plan and online educational continuity. Objective: The study aimed to know the changes in teaching processes due to the COVID-19 health emergency. Method. The study was based on a mixed descriptive design that integrated quantitative and qualitative data. The information was collected through a self-administered digital survey applied to 470 teachers between May and June 2020; they were working at different teaching levels, from early education to university. Results. Even in a country like Uruguay with high educational connectivity, the suspension of in-person classes due to COVID-19 caused tensions and challenges at the work, pedagogy, and technology levels. There was a negative impact on occupational health and teacher stress. In addition, problems of low appropriation and pedagogical use of technologies the teaching staff experienced were registered. Discussion. One of the significant challenges the teaching staff pointed out is constructing new pedagogical formats to improve educational results with equity.
\end{abstract}

Keywords: Virtual learning; teaching practices; pandemics; teaching profession.

\begin{abstract}
Resumo:
Introdução. Este artigo apresenta resultados para o caso do Uruguai de uma investigação internacional que buscou a percepção dos professores sobre o ensino remoto de emergência. A temática foi investigada em um contexto nacional que apresenta duas características distintivas: o acesso universal a recursos tecnológicos para o ensino devido à cobertura nacional do Plan Ceibal e a continuidade educacional em linha. Objetivo. O objetivo de estudo foi conhecer as mudanças geradas nos processos de ensino devido à emergência de saúde causada pela COVID-19. Metodo. O estudo foi baseado em um desenho descritivo misto que integrou dados quantitativos e qualitativos. A informação foi coletada por meio de uma pesquisa digital autoadministrada aplicada a 470 professores entre maio e junho de 2020 . Foram analisadas as respostas de professores que atuam em diferentes níveis de ensino, desde a formação inicial até o ensino superior. Resultados. Mesmo em um país como o Uruguai com alta conectividade educacional, a suspensão das aulas presenciais devido a COVID-19 causou tensões e desafios no ambiente de trabalho, pedagógico e tecnológico. Houve impacto negativo na saúde ocupacional e no estresse docente. Além disso, foram registrados problemas de baixa apropriação e uso pedagógico das tecnologias pelos professores. Discussão. Um dos grandes desafios apontados pelos professores referese à construção de novos formatos pedagógicos para melhorar os resultados educacionais com equidade.
\end{abstract}

Palavras-chave: Aprendizagem virtual; práticas de ensino; pandemia; profissão docente. 


\section{Introducción}

El sector educativo no estaba preparado para una disrupción como la que ha traído consigo la pandemia de la COVID-19. Los cierres de las instituciones educativas para frenar la pandemia llevaron a un despliegue acelerado de intervenciones de educación a distancia para asegurar la continuidad pedagógica. Los obstáculos han sido múltiples, desde pedagógicos y tecnológicos hasta los cambios en las condiciones laborales del personal docente (Pedró, 2020).

El cierre total o parcial de los centros educativos exige una estrategia intencional y efectiva para garantizar la oportunidad de aprender en tiempos de pandemia y evitar graves pérdidas de aprendizaje para el estudiantado (Reimers y Schleicher, 2020). Y en este escenario, es esperable que países como Uruguay con alta conectividad educativa, dispongan de mejores condiciones para atender la crisis sanitaria en las escuelas. Recordemos que el país impulsa la inclusión digital de tecnologías educativas, desde hace casi quince años, a través del Plan Ceibal. Este plan fue creado en el año 2007 con el fin de promover políticas de inclusión e igualdad de oportunidades mediante tecnologías. Los niños, las niñas y jóvenes estudiantes uruguayos tienen la posibilidad de acceder a computadoras personales, que cuentan con conexión a Internet gratuita a través de su centro educativo. Pero, además, el Plan Ceibal impulsa el desarrollo profesional docente, así como la creación de programas y recursos didácticos (Plan Ceibal, s.f.). Sin embargo, y a pesar del esfuerzo realizado en los últimos 15 años en materia de políticas de desarrollo profesional docente, el uso con sentido pedagógico de las TIC todavía es incipiente (Cobo y Montaldo, 2018) y es probablemente una de las mayores dificultades que ha registrado la educación remota de emergencia luego de la COVID-19.

El análisis que presentamos en este artículo se enmarca en un proyecto de investigación comparada y de carácter internacional llevado adelante por un equipo de la Universidad ORT Uruguay. El estudio fue diseñado y promovido por investigadores e investigadoras de la Universidad Da Vinci de México en el cual ha participado personal académico de Brasil, Chile, Chipre, Colombia, Dinamarca, Nigeria, Perú, Reino Unido y Uruguay. El objetivo fue contribuir a la comprensión de las percepciones del personal docente ante los cambios educativos por la cancelación de las clases presenciales. El análisis se focalizó en los resultados primarios obtenidos en Uruguay y en particular en el personal docente de todos los niveles educativos, desde inicial a la universidad. Su situación se ha caracterizado por una sobrecarga de demanda de trabajo durante la pandemia, especialmente a nivel cognitivo, cuantitativo y emocional, factores que inciden en el bienestar docente y en las capacidades de adaptabilidad (Cardozo et al., 2020; Sosa y Ambrosi, 2021; Canal oficial de la Unidad de Tecnología Educativa del Consejo de Formación en Educación UATE-CFE, 2020).

El estudio busca responder a dos interrogantes principales:

- ¿Cuáles son las percepciones del personal docente acerca de la enseñanza remota de emergencia durante el cierre de los centros educativos? 
https://doi.org/10.15359/ree.26-1.4

https://www.revistas.una.ac.cr/index.php/educare

educare@una.ac.cr

- ¿Cuáles son las percepciones del personal docente acerca de las modalidades para el desarrollo de la enseñanza remota de emergencia durante el cierre de los centros educativos?

También, el estudio indagó sobre los principales retos pedagógicos percibidos por el personal docente.

\section{Incidencia de la pandemia en educación}

Durante el primer semestre de 2020 diversos estudios procuraron describir y analizar la incidencia del cierre de las escuelas a causa de la COVID-19. Encuestas y entrevistas fueron realizadas en los cinco continentes a directivos, docentes, y al propio estudiantado y sus familias. Entre esas investigaciones se destacan las de Lee, 2020; Reimers y Schleicher, 2020; United Nations Educational, Scientific and Cultural Organization [UNESCO], 2020.

Revistas indexadas de prestigio han dedicado dossiers especiales a la temática de la pandemia durante el 1er semestre 2020. Así, a nivel internacional, el Journal Asia-Pacific Journal of Teacher Education (Vol. 48, №3, 2020) consagra una edición especial a la temática de Teaching and teacher education in the time of COVID-19. Los seis artículos del número analizan los cambios en la vida profesional del personal docente y en particular el de Li (2020) examina a partir de una muestra de 426 docentes en cinco universidades chinas, la relación entre el apoyo institucional recibido y el compromiso afectivo del personal docente.

En el nivel iberoamericano varias publicaciones han dedicado sus números a estudios y análisis vinculados con la situación generada por la COVID-19, tal fue el caso de la Revista Internacional de Educación para la Justicia Social (Vol. 9, № 3, 2020) publicada por la Universidad Autónoma de Madrid que consagra un número extraordinario a las Consecuencias del Cierre de Escuelas por el COVID-19 en las Desigualdades Educativas. El artículo de Cáceres-Muñoz et al. (2020) ofrece una "perspectiva internacional sobre el proceso de cierre de escuelas y sus consecuencias educativo-sociales" (p. 199), a partir de entrevistas a informantes claves, pertenecientes a 23 países, de cuatro continentes diferentes.

Los hallazgos permiten observar una gran diversidad en las formas de enfrentar la pandemia, alineadas a lo que Hodges et al. (2020), denominaron enseñanza remota de emergencia. Aunque la evidencia es aún reciente, los resultados de estudios realizados durante el año 2020 permiten identificar consecuencias similares para las poblaciones más vulnerables, debido al incremento de la desigualdad educativa y social generada por la COVID-19 (CáceresMuñoz et al., 2020). Lo que parece una constante en los estudios citados, es que la situación sanitaria ha desencadenado cambios en la configuración de la enseñanza y del aprendizaje tal como la conocíamos hasta el momento. La COVID-19 interpela a los modelos actuales de enseñanza y marca la transición hacia un nuevo paradigma del trabajo docente.

4

Denise Vaillant, Eduardo Rodríguez-Zidán y Mariela Questa-Torterolo

Los artículos de la Revista Electrónica Educare del Centro de Investigación y Docencia en Educación de la Universidad Nacional, Costa Rica, se comparten bajo términos de la Licencia Creative Commons: Reconocimiento, No Comercial, Sin Obra Derivada 3.0 Costa Rica. Las autorizaciones adicionales a las aquí delimitadas se pueden obtener en el correo: educare@una.cr 


\section{Educación y tecnología en Uruguay antes de la COVID-19}

La estrategia en Uruguay para enfrentar la crisis de la COVID-19 fue suspender las clases presenciales entre marzo y junio del año 2020, apostando a la educación a distancia. La continuidad de los aprendizajes en la educación obligatoria fue asegurada por el Plan Ceibal, implementado en el país desde hace casi quince años. Sin embargo, la incidencia del Plan en las prácticas docentes ha sido diversa. En educación primaria parecería que los maestros y las maestras han hecho un uso pedagógico más extendido de la tecnología. Así, un estudio confirmó que el uso de la plataforma adaptativa de matemática por el personal docente tuvo efectos positivos en los aprendizajes de niñas y niños (Perera y Aboal, 2017).

En educación media el panorama es menos alentador y los desafíos parecen ser mayores. En 2012, un estudio coordinado por Vaillant (2012) puso en evidencia el escaso uso pedagógico de las tecnologías por parte del personal docente y del estudiantado en el aula de Matemáticas. Por su parte Lamschtein (2018) concluyó que “las tecnologías no se habían incluido en las prácticas de enseñanza y aprendizaje, sino que los estudiantes, casi en su totalidad, las empleaban para resolver las tareas domiciliarias, además de utilizarlas con fines recreativos" (p. 8).

También en educación media, reportes de investigación dan cuenta del escaso uso pedagógico de las computadoras personales entregadas por Ceibal. Además, que los teléfonos celulares estarían desplazando a los dispositivos del modelo uno a uno como recursos de apoyo a la docencia (Cabrera Borges et al., 2018-2019). Otro informe reciente, evidencia que un 50\% de los profesores de matemática del 1er ciclo de educación media utiliza la Plataforma Adaptativa de Matemática (PAM) con frecuencia alta mientras que en el 50\% restante el uso es bajo o nulo (Vaillant et al., 2020).

En cuanto al nivel de formación docente, el uso de recursos pedagógicos era moderado y bajo antes de la COVID-19 tal como lo señala una investigación referida a la plataforma CREA que constituye un entorno virtual de aprendizaje para gestionar cursos, crear o compartir materiales didácticos. El mencionado estudio revela que solamente el 15\% de personas formadoras de docentes utilizaba la plataforma (Rodríguez Zidán et al., 2017).

En lo que respecta al nivel universitario, Uruguay ha mantenido tradicionalmente un monopolio centralizado y urbano y una concepción presencialista de la educación con escasa cobertura fuera de la capital del país (Rama, 2020). Antes de la COVID-19, un reporte de investigación halló que los entornos virtuales de aprendizaje en las facultades de Química e Ingeniería de la Universidad de la República eran utilizados fundamentalmente como recursos de apoyo para las actividades presenciales de grado (Bühl, 2013).

Tal lo señalado en diversos informes del Instituto Nacional de Evaluación Educativa (Alonso et al., 2018; Alonso et al., 2020) a pesar de las políticas tecnológicas y docentes implementadas 
https://doi.org/10.15359/ree.26-1.4

https://www.revistas.una.ac.cr/index.php/educare

educare@una.ac.cr

por el Plan Ceibal en los últimos años, el panorama previo a la pandemia en Uruguay evidenciaba un uso pedagógico limitado de dispositivos y recursos digitales en apoyo a la enseñanza, en especial en educación media y formación docente.

\section{Apuntes metodológicos}

A partir de las dos interrogantes principales se ha buscado indagar más específicamente en los cambios percibidos por el personal docente en sus condiciones de trabajo; las principales prácticas docentes implementadas para atender la situación de emergencia; las competencias TIC, preparación y acceso a Internet por parte del personal docente y análisis de tópicos cualitativos emergentes vinculados con los desafíos y retos que plantea la enseñanza remota a las prácticas docentes.

Para responder a las preguntas de investigación, se adoptó un enfoque mixto, con énfasis descriptivo, no experimental transaccional tipo survey. El abordaje cuantitativo se desarrolló a partir una encuesta en línea autoadministrada que se complementó con el análisis de tópicos cualitativos emergentes.

La evidencia fue colectada durante los meses de mayo-junio 2020 mediante una encuesta a docentes de todos los niveles educativos desde educación inicial al nivel universitario distribuida a través de redes sociales (Twitter y Linkedln y redes profesionales). Si bien la encuesta no es representativa en todas las variables consideradas, se buscó llegar a una diversidad de encuestados que reflejaran una diversidad de perspectivas y posiciones en el sector educativo. Los datos para el estudio se obtuvieron de una encuesta online de tipo auto reporte. El tipo de muestra es no probabilística, transaccional y por conveniencia. Y como se indicará más adelante la consistencia interna fue medida con el Alpha de Cronbach.

El universo de análisis se constituyó de un total de 470 docentes ( $82 \%$ género femenino) con una media de edad de 44 años, dispersión de 10.29, (mínimo 20 y máximo 72 años) y experiencia profesional de 10 años o más en el desempeño del rol docente en el nivel respectivo. Respecto a la distribución por subsistema, el $50.8 \%$ se desempeña en educación secundaria, $27.5 \%$ en primaria, $8,7 \%$ en formación docente y $11.2 \%$ en la universidad. Tanto la distribución de la muestra por tipo de sector (70.7 \% público y $29.3 \%$ privado) como región (91\% urbano) presentan datos representativos de la población docente general del sistema público (Administración Nacional de Educación Pública [ANEP], 2020a).

Luego de culminar la etapa de adaptación y validación por el equipo de personas investigadoras del estudio que se presenta en este artículo, el instrumento aplicado quedó constituido por 39 reactivos, 6 escalas y dos preguntas abiertas. El análisis focaliza en algunas de las dimensiones y secciones del cuestionario, tal como se observa en la Tabla 1. 
https://doi.org/10.15359/ree.26-1.4

Tabla 1: Dimensiones del objeto de estudio, variables seleccionadas y tipos de preguntas

\begin{tabular}{|c|c|c|c|}
\hline Dimensiones & Variables & Indicadores & Tipo de pregunta \\
\hline Sociodemográfica & $\begin{array}{l}\text { Edad, genero, tipo de rol, } \\
\text { subsistema, sector educativo, } \\
\text { ubicación geográfica }\end{array}$ & $\begin{array}{l}\text { Edad de la persona participante en años, } \\
\text { género, ocupación actual, nivel educativo } \\
\text { en el que enseña, lugar del centro }\end{array}$ & $\begin{array}{l}\text { Cuantitativa y } \\
\text { Politómicas }\end{array}$ \\
\hline Contexto laboral & Condiciones de trabajo & $\begin{array}{l}\text { Cambios producidos por la pandemia; } \\
\text { espacio de trabajo en el hogar; acceso } \\
\text { a tecnologías, apoyos institucionales; } \\
\text { directivas organizacionales para } \\
\text { modificación de cursos; productividad } \\
\text { laboral; apoyos en el hogar y entorno } \\
\text { trabajo desde mi hogar. }\end{array}$ & $\begin{array}{l}\text { Escalas Likert y } \\
\text { Politómicas }\end{array}$ \\
\hline Pedagógica & $\begin{array}{l}\text { Prácticas docentes, } \\
\text { enfoques pedagógicos y } \\
\text { expectativas }\end{array}$ & $\begin{array}{l}\text { Prácticas docentes realizadas durante } \\
\text { la pandemia; cambios producidos por } \\
\text { la docencia remota de emergencia; } \\
\text { incidencia de los cambios en las prácticas } \\
\text { docentes; conocimiento de enfoques } \\
\text { pedagógicos para el aprendizaje en línea; } \\
\text { disposición a experimentar cambios en } \\
\text { las prácticas; confianza en la capacidad de } \\
\text { enseñar en línea. }\end{array}$ & $\begin{array}{l}\text { Respuesta } \\
\text { múltiple, Escala } \\
\text { Likert }\end{array}$ \\
\hline Tecnológica & $\begin{array}{l}\text { Competencias TIC, } \\
\text { preparación y acceso a } \\
\text { Internet }\end{array}$ & $\begin{array}{l}\text { Competencia para el uso de tecnologías } \\
\text { educativas; percepción sobre la } \\
\text { preparación para la enseñanza en línea; } \\
\text { nivel de conexión a Internet en el hogar. }\end{array}$ & $\begin{array}{l}\text { Escalas Likert, } \\
\text { Politómicas }\end{array}$ \\
\hline Retos & Desafíos & $\begin{array}{l}\text { ¿Cuál es el principal reto que enfrenta } \\
\text { en su práctica docente durante esta } \\
\text { pandemia de la COVID-19? }\end{array}$ & Abierta \\
\hline
\end{tabular}

Nota: Elaboración propia.

En las tres escalas consideradas (dimensión laboral, pedagógica y tecnológica), los ítems están fuertemente correlacionados y la fiabilidad estadística es muy buena y excelente. Los valores observados dan cuenta de la consistencia interna y, en consecuencia, miden un mismo constructo teórico (valores de Alpha de Cronbach de 0,829, 0,890 y 0,910 para cada escala respectivamente). 
https://doi.org/10.15359/ree.26-1.4

https://www.revistas.una.ac.cr/index.php/educare

educare@una.ac.cr

Se empleó una estrategia de análisis que integró el estudio descriptivo y contraste de hipótesis con análisis cualitativo. Para el primer enfoque, se aplicaron análisis de medidas de tendencia central, dispersión, frecuencia. Para el cálculo estadístico se utilizó el software SPSS 23. En una segunda fase se codificaron las respuestas abiertas del instrumento, mediante el procedimiento de construcción de categorías emergentes y ad hoc con el apoyo de Atlas.ti versión 8 . En esta fase se realizó un análisis temático de tópicos emergentes.

El plan de análisis partió de la información obtenida por las variables descriptivas seleccionadas, para posteriormente analizar las escalas de opinión en cada una de las tres dimensiones del objeto de estudio (laboral, pedagógica y tecnológica) y observar el grado de incidencia de la variable subsistema y tipo de institución en las variables consideras relevantes. Para la fase de análisis de los datos que surgen de las preguntas abiertas del cuestionario, se elaboró un conjunto de categorías interpretativas y explicativas, agrupando y reagrupando los hallazgos siguiendo el procedimiento propuesto por el método comparativo (Miles y Huberman, 1994).

En primer lugar, los resultados del estudio se organizaron según un enfoque cuantitativo con base en los dos constructos identificados a partir del análisis estadístico de las preguntas seleccionadas para este informe. De acuerdo con este procedimiento, los datos se agruparon según variables referidas a la dimensión laboral y pedagógica de la enseñanza. Asimismo, se efectuó un estudio descriptivo y de asociación de variables ligadas a las principales prácticas pedagógicas implementadas, observando si existen diferencias significativas según el nivel educativo de desempeño docente. En segundo lugar, se agruparon los resultados del abordaje cualitativo, y se identificaron los retos que representa la enseñanza remota para la población estudiada. Los hallazgos cualitativos posibilitaron una mayor comprensión y análisis del significado que le atribuye el personal docente uruguayo a los principales retos y desafíos que enfrentaron en sus clases virtuales.

\section{Resultados}

En los párrafos que siguen se busca responder a las preguntas de investigación. Los resultados se agrupan en torno a las percepciones de los docentes acerca de la enseñanza remota, tanto en lo que hace a la dimensión laboral como la pedagógica. También se presenta evidencia acerca de los retos planteados.

\section{La dimensión laboral}

Tal como analizaremos en los párrafos que siguen, los resultados del estudio realizado en Uruguay son congruentes con las evidencias internacionales y regionales que muestran que uno de los efectos provocados por el confinamiento fue el impacto en las condiciones laborales de la docencia. La docencia de emergencia significó un aumento del estrés y la presión hacia 
el personal docente como en otros sistemas educativos. Así, según un estudio en realizado en España, la pandemia de la COVID-19 ha supuesto para la educación una auténtica prueba de estrés (Luengo y Manso, 2020). En América Latina, la situación del cierre de escuelas y las consecuencias del confinamiento voluntario u obligatorio, enfrentó al personal docente a vivir situaciones de desgaste emocional, agobio y estrés (UNESCO, 2020).

El estudio realizado en Uruguay muestra que un 33\% del personal docente se manifestó estresado durante el período de confinamiento. Asimismo, dos de cada tres docentes (67\%) expresaron sentimientos encontrados asociados, por un lado, a la ansiedad e incertidumbre provocada por la educación a distancia y la desvinculación con los estudiantes, pero por otro, al surgimiento del deseo de aprender nuevas metodologías y herramientas virtuales para mejorar sus prácticas de uso de TIC para la enseñanza.

Las respuestas de las personas docentes que surgen de los ítems abiertos del cuestionario que se presentan en la Tabla 2 son concluyentes respecto a sentimientos de preocupación, angustia, a la búsqueda de equilibrio emocional y el logro de aprendizajes colectivos. También los relatos evidencian los desafíos que implica construir un modelo de enseñanza que articule la presencialidad y la virtualidad de manera eficiente, productiva y a largo plazo.

Otro conjunto de indicadores relevados pertenecientes al contexto laboral y condiciones de trabajo refiere a un acceso a Internet de calidad para la docencia, el apoyo familiar, las condiciones espaciales en el hogar para la educación en línea, así como la intensidad percibida acerca del trabajo docente durante el confinamiento (ver Tabla 1).

Un alto porcentaje del personal docente consultado declaró que tiene acceso a Internet de banda ancha en su hogar (84\%) y además contó con el apoyo de la familia. El $80 \%$ del personal docente participante del estudio señaló estar de acuerdo o totalmente de acuerdo con el apoyo recibido por sus familiares o personas que vivían en su hogar para el desarrollo de los cursos en línea durante el periodo de emergencia.

En lo referido a las condiciones para el desempeño del rol docente durante la pandemia, la mayoría de los maestros, las maestras y profesorado (ver Tabla 3) tuvo acceso a un espacio en su hogar para el desarrollo de la enseñanza en línea (60\%). No obstante, resulta preocupante que uno de cada cuatro docentes manifiesta no disponer de las condiciones espaciales mínimas en su domicilio para el trabajo a distancia. Aunque este porcentaje aumentaría significativamente en función de la estratificación social tal lo señala la encuesta a docentes realizada por las autoridades de la educación en el mes de julio (ANEP, 2020b). 
https://doi.org/10.15359/ree.26-1.4

https://www.revistas.una.ac.cr/index.php/educare

educare@una.ac.cr

Tabla 2: ¿Cómo se sintió el personal docente uruguayo en relación con los cambios educativos derivados de la pandemia de la COVID-19?

Sujeto
Profesor del ciclo básico público
Profesora de educación media
básica privada

Unidad de significado

intentando equilibrar la adrenalina doméstica (C_132)

He pasado por todos los estados: primero estresada, luego tranquila, y finalmente emocionada por la novedad de los Webinar de las cuales participo todos los jueves, dado que se presentan contenidos muy ricos para incorporar a la nueva realidad educativa $\left(C_{-} 176\right)$

Profesor de ciclo básico público Muy positivo en ciclo básico para los estudiantes. Los obligó a aprender a usar las tecnologías CREA (y por lo tanto los preparara para el uso de cualquiera plataforma). En cuanto a la gestión docente, la capacidad de registro de las plataformas asegura que no se produzca ruido en la entrega de tareas. Por ruido me refiero a situaciones de conflicto como, por ejemplo, estudiantes que dicen que hicieron las tareas, pero CREA no las registró y otras (C_204)

Profesora de 2 do ciclo de

En realidad, fue un proceso. Del estrés total hacia la tranquilidad a partir de la educación básica privada organización (C_309)

Profesora de 1 y 2 do ciclo de educación privada y pública

Estresado, preocupado, angustiado y con muchísimas ganas de llegar a los estudiantes que aún no pude comunicarme, por diversos motivos (falta de herramientas y condiciones, por ejemplo) (C_50)

Profesora de universidad pública Recién estamos procesando el impacto de tantos cambios. La incertidumbre del contexto actual y futuro me plantea dudas sobre el desempeño acertado de mi propio rol docente; fundamentalmente por la preocupación sobre la situación de los estudiantes ante la ausencia de la figura del profesor. Además de estar consciente que tengo que innovar en las formas de mantener un contacto estrecho con los estudiantes en ámbitos de masividad (C_341)

Profesora universidad privada Los cambios educativos se suman al impacto emocional del confinamiento, a tiempos complejos vitales propios a manejar fuera de lo profesional (C_397)

Profesora de primaria pública

No existe un compromiso similar en los colectivos docentes. El resultado a obtener puede ser muy disímil (C_207)

Profesor de 2do ciclo de

Lo que he aprendido no debe quedar olvidado. Lo mejor sería hacer un mix de secundaria presencialidad y virtualidad. esto permitiría utilizar el tiempo de aula para otros aprendizajes que son obligatoriamente presenciales, como actividad física (C_57)

Nota: Elaboración propia.

10 Denise Vaillant, Eduardo Rodríguez-Zidán y Mariela Questa-Torterolo

Los artículos de la Revista Electrónica Educare del Centro de Investigación y Docencia en Educación de la Universidad Nacional, Costa Rica, se comparten bajo términos de la Licencia Creative Commons: Reconocimiento, No Comercial, Sin Obra Derivada 3.0 Costa Rica. Las autorizaciones adicionales a las aquí delimitadas se pueden obtener en el correo: educare@una.cr 
https://doi.org/10.15359/ree.26-1.4

Tabla 3: ¿Qué tan de acuerdo está con las siguientes afirmaciones referidas a su contexto laboral?

\begin{tabular}{|c|c|c|c|c|c|c|c|c|}
\hline \multirow{2}{*}{ Pregunta } & \multirow{2}{*}{$\mathrm{N}$} & \multicolumn{5}{|c|}{$\%$} & \multirow{2}{*}{ Media } & \multirow{2}{*}{ DT } \\
\hline & & TD & D & $\mathrm{N}$ & $A$ & TA & & \\
\hline $\begin{array}{l}\text { Cuento con un espacio en mi hogar adecua- } \\
\text { do para realizar mi docencia en línea }\end{array}$ & 470 & 8.7 & 18.3 & 13.0 & 30.1 & 29.9 & 3,54 & 1,319 \\
\hline $\begin{array}{l}\text { Tengo acceso a las tecnologías que necesito } \\
\text { usar para modificar mis clases }\end{array}$ & 470 & 5.1 & 7.2 & 14.3 & 39.4 & 33.9 & 3,90 & 1,107 \\
\hline $\begin{array}{l}\text { Mi productividad se ha incrementado desde } \\
\text { que trabajo desde mi hogar }\end{array}$ & 470 & 7.9 & 18.8 & 29.6 & 26.9 & 16.8 & 3,26 & 1,175 \\
\hline $\begin{array}{l}\text { Mi institución educativa cuenta con un plan } \\
\text { claro para apoyar las modificaciones que se } \\
\text { deben realizar en los cursos }\end{array}$ & 470 & 16.2 & 21.5 & 27.5 & 23.5 & 11.3 & 2,92 & 1,24 \\
\hline
\end{tabular}

TD: Totalmente en desacuerdo, D: En desacuerdo, N: Ni acuerdo ni en desacuerdo, A: De acuerdo, TA: Totalmente de acuerdo.

Nota: Elaboración propia.

Otro indicador elocuente de la intensidad del trabajo en línea refiere a la productividad. Según las percepciones del personal docente, la educación a distancia desde el hogar significó un aumento de la productividad docente para un $43.7 \%$ de los encuestados. Por último, tal como se indica en la Tabla 4, una proporción muy baja de docentes $(34,8 \%)$ está de acuerdo o totalmente de acuerdo con la afirmación de que su institución contaba con un plan claro de apoyo para el desempeño de la docencia en línea de emergencia. Este dato pone en evidencia la necesidad de fortalecer el liderazgo institucional para promover nuevos modelos de gestión eficiente de las TIC y de "involucrar a los equipos de conducción de los sistemas educativos en los procesos de integración de las políticas digitales" (Lugo y Delgado, 2020, p. 22).

\section{La dimensión pedagógica}

En segundo lugar, en relación con las prácticas educativas realizadas durante el periodo de la emergencia sanitaria la gran mayoría del personal docente consultado (83\%), e independientemente del sector educativo o el nivel donde se desempeñan, declaró que su estrategia principal fue trasladar los contenidos y las actividades presenciales de sus cursos a la modalidad de trabajo docente en línea. En efecto, los datos de la Tabla 4 revelan que el principal cambio impulsado por el personal docente fue la transformación y adaptación del formato presencial de enseñanza a la educación virtual. 
https://doi.org/10.15359/ree.26-1.4

https://www.revistas.una.ac.cr/index.php/educare

educare@una.ac.cr

Los hallazgos muestran que las prácticas docentes durante la pandemia se debieron adaptar a un modelo de enseñanza totalmente no presencial donde el acceso (por parte de docentes, estudiantes y familias) a tecnologías e Internet de calidad se constituye como elementos esenciales que representan los principales desafíos para mantener los procesos de enseñanza y aprendizaje a pesar de la pandemia.

Tabla 4: Prácticas pedagógicas desarrolladas durante la pandemia según nivel educativo

\begin{tabular}{|c|c|c|c|c|c|}
\hline \multirow[b]{2}{*}{ Pregunta } & \multicolumn{4}{|c|}{ Nivel educativo } & \multirow[b]{2}{*}{ Promedio } \\
\hline & Primaria & Secundaria & Formación Docente & Universidad & \\
\hline $\begin{array}{l}\text { Desarrollo de Enseñanza Remota de } \\
\text { Emergencia }\end{array}$ & $44 \%$ & $47 \%$ & $32 \%(*)$ & $51 \%$ & $43.5 \%$ \\
\hline Rediseño pedagógico de cursos & $73.1 \%$ & $77 \%$ & $76 \%$ & $79 \%$ & $76.2 \%$ \\
\hline $\begin{array}{l}\text { Continuación de sus prácticas } \\
\text { docentes de manera regular }\end{array}$ & $27.7 \%$ & $27.7 \%$ & $26.4 \%$ & $26.4 \%$ & $27 \%$ \\
\hline Suspensión de actividades & $3.1 \%$ & $4.2 \%$ & $2.2 \%$ & $3.8 \%$ & $3.3 \%$ \\
\hline $\begin{array}{l}\text { Subida de materiales de curso a un } \\
\text { repositorio en línea }\end{array}$ & $40 \%$ & $46 \%$ & $52 \%$ & $59 \%$ & $49.2 \%$ \\
\hline $\begin{array}{l}\text { Solicitud a los estudiantes de } \\
\text { realización de actividades por su } \\
\text { cuenta sin apoyo en línea }\end{array}$ & $3.1 \%$ & $4.6 \%$ & $8.7 \%$ & $5.7 \%$ & $5.5 \%$ \\
\hline $\begin{array}{l}\text { Cambio de las sesiones presenciales } \\
\text { por videoconferencia }\end{array}$ & $70 \%$ & $64 \%$ & $54 \%(* *)$ & $72 \%$ & $65 \%$ \\
\hline
\end{tabular}

${ }^{*}$ ) Diferencia significativa (p.0.025, valor Chi Cuadrado 23,298).

(**) Diferencia significativa (p.0.049, Chi cuadrado 12,398).

Nota: Elaboración propia.

El76\% del personal docente manifestó que tuvo que rediseñar pedagógicamenteloscursos para adaptar contenidos y actividades a un formato digital, incluyendo actividades sincrónicas o asincrónicas. En particular, el cambio de las sesiones presenciales por videoconferencias (por ejemplo, usando Zoom) fue otro cambio destacado por la mayoría del personal docente consultado (65\%). Asimismo, el personal docente señaló la importancia de subir materiales del curso a repositorios en línea y plataformas de contenidos digitales (47\%).

Es de destacar que el $96 \%$ del personal docente encuestado no suspendieron sus clases y que únicamente el $4 \%$ de la muestra indicó que solicitó a sus estudiantes realizar actividades por su cuenta sin contar con apoyo en línea. Cabe señalar que no todo el personal docente que continuó sus cursos transformó sus formas habituales de trabajo presencial: el $27 \%$ de la población consultada, aunque no suspendió las clases, continuó sin modificar su forma de trabajo previa a la llegada de la COVID-19. 
Si bien las evidencias relevadas permiten inferir el esfuerzo realizado por el personal docente para sostener la enseñanza en modalidad virtual y en todos los subsistemas educativos (desde educación primaria hasta la universidad) no todo el personal docente acuerda en definir los cambios realizados como una enseñanza remota de emergencia. Cuando se les consultó respecto a si los cambios impulsados en sus clases constituyeron una enseñanza remota de emergencia, el $43.5 \%$ respondió afirmativamente, con diferencias estadísticamente significativas (p.0.025, valor Chi Cuadrado 23,298) entre subsistemas: el porcentaje desciende a $32 \%$ entre las personas formadoras de docentes y aumenta a $51 \%$ entre las personas universitarias).

Por último, dos aspectos merecen ser comentados como hallazgos relevantes para profundizar en la discusión sobre políticas educativas en el contexto de pandemia. En primer lugar, el estudio relevó la opinión del personal docente acerca de la incidencia de dos aspectos de la dimensión subjetiva en las prácticas docentes. Diversos reportes de investigación han identificado la incidencia de la promoción de la confianza y la disposición del personal docente para impulsar transformaciones de la enseñanza como dos elementos claves para sostener procesos de cambio y mejora especialmente en el aumento del saber profesional docente eincremento en los aprendizajes del alumnado (Bolívar et al., 2013). Respecto a estos dos factores, en la Tabla 5 se observa que la gran mayoría del personal docente ha tenido sentimientos de confianza para la enseñanza en línea (73.8\%) y una proporción mayor aún (84.7\%) expresa su disposición a experimentar para modificar las prácticas docentes. Este hallazgo da cuenta de la existencia de condiciones subjetivas y de expectativas de cambio muy favorables a la participación del personal docente en procesos de formación de nuevos formatos de enseñanza híbrida o educación combinada que integren lo mejor de la clase presencial con lo mejor de la educación en línea.

Tabla 5: ¿Qué tan de acuerdo está con las siguientes afirmaciones?

\begin{tabular}{|c|c|c|c|c|c|c|c|c|}
\hline \multirow[t]{2}{*}{ Pregunta } & \multirow[t]{2}{*}{$\mathbf{N}$} & \multicolumn{5}{|c|}{$\%$} & \multirow[t]{2}{*}{ Media } & \multirow[t]{2}{*}{ DT } \\
\hline & & TD & D & $\mathrm{N}$ & A & TA & & \\
\hline $\begin{array}{l}\text { Conozco lo suficientes sobre enfoques } \\
\text { pedagógicos apropiados para apoyar el } \\
\text { aprendizaje en línea }\end{array}$ & 470 & 4.9 & 20.7 & 24.7 & 37.3 & 12.4 & 3,32 & 1,083 \\
\hline $\begin{array}{l}\text { Estoy dispuesto(a) a experimentar para } \\
\text { modificar mis prácticas docentes }\end{array}$ & 470 & 3.0 & 3.6 & 8.7 & 35.4 & 49.3 & 4,24 & ,968 \\
\hline $\begin{array}{l}\text { Tengo confianza en mi capacidad para } \\
\text { enseñar en línea }\end{array}$ & 470 & 1.3 & 6.8 & 18.1 & 47.8 & 26.0 & 3,90 & 907 \\
\hline
\end{tabular}

TD:Totalmente en desacuerdo, D: En desacuerdo, N: Ni de acuerdo ni en desacuerdo, A: De acuerdo, TA:Totalmente de acuerdo.

Nota: Elaboración propia. 
https://doi.org/10.15359/ree.26-1.4

https://www.revistas.una.ac.cr/index.php/educare

educare@una.ac.cr

Asimismo, fue de interés conocer el grado de conocimiento que tiene el personal docente de la muestra respecto a los enfoques pedagógicos para la virtualidad. A pesar de las buenas condiciones de desarrollo de infraestructura tecnológica yacceso a Internet queelUruguay dispone desde la creación temprana del Plan Ceibal en el año 2007 (acceso a tecnologías, plataformas gratuitas de aprendizaje en línea e Internet de banda ancha) y subjetivas (altas expectativas y autoconfianza para experimentar nuevos formatos pedagógicos) y de las políticas impulsadas por Ceibal para la formación docente en nuevas prácticas pedagógicas (Plan Ceibal, 2020), la mitad del profesorado consultado (49.7\%) desconoce aún cuáles son los enfoques pedagógicos específicos que permiten desarrollar la enseñanza virtual con herramienticas digitales y a distancia (Tabla 5). Este dato es relevante por su implicancia para el diseño de políticas, en especial porque también hemos encontrado que la mayoría del personal docente ha sentido que la institución educativa donde trabajan no cuenta con un plan claro de apoyo al personal docente (65.2\%).

\section{Los retos de la enseñanza remota de emergencia}

En relación con las percepciones del personal docente respecto a los retos que conlleva la enseñanza remota de emergencia, estos fueron agrupados en cuatro dimensiones: laboral, pedagógica, tecnológica y emocional. Dentro de cada dimensión, se identificaron subcategorías determinadas para la codificación de las respuestas. En el caso de los retos laborales las categorías emergentes fueron: i) organización del trabajo, ii) modalidad de trabajo, y iii) formación previa o en servicio. Asociadas a los retos pedagógicos, las categorías identificadas fueron: i) acompañamiento, ii) aprendizajes, iii) didáctica, iv) evaluación, y v) motivación y participación. En cuanto a los retos tecnológicos, la categoría engloba i) infraestructura tecnológica (disponibilidad de conectividad y dispositivos competencias), y ii) tiempo que insume la enseñanza remota de emergencia mediada por las tecnologías. Por último, y en relación con los retos emocionales se identificaron como categorías: i) comunicación y vínculos, ii) cuestiones emocionales propiamente dichas, y iii) vínculo y apoyo de las familias.

Cabe destacar que se lograron trazar asociaciones entre los diversos códigos. Por ejemplo, en la categoría retos laborales, las respuestas relacionadas a la formación previa o en servicio guardan relación con los retos pedagógicos que identifica el personal docente. En el caso de la motivación y la participación del estudiantado en las propuestas pedagógicas, las respuestas indican también los retos vinculares que identifica el personal docente. Si se observa la categoría tiempo, las personas docentes identifican un reto tanto a nivel laboral, por lo que insume la enseñanza remota de emergencia, como a nivel pedagógico, marcando las dificultades para adaptar la propuesta remota.

Al analizar las evidencias según nivel educativo en que enseña el personal docente, se logró categorizar los retos según las frecuencias observadas. Sin embargo, en el conteo general de frecuencias asociadas a los retos laborales (organización del trabajo, modalidad de trabajo, 
https://doi.org/10.15359/ree.26-1.4

y formación previa o en servicio) coinciden en ser las menos mencionadas como desafíos en el contexto de enseñanza remota de emergencia, en todos los niveles educativos analizados.

En el grupo de docentes pertenecientes al nivel de educación inicial y primaria, lograr la motivación y la participación de los estudiantes en la propuesta remota, ocupa el primer lugar como reto, junto con las cuestiones referidas a la disponibilidad de tecnologías. Asimismo, resulta un desafío, adaptar el acompañamiento de la población estudiantil y las actividades mediadas por las tecnologías que se proponen. Por otra parte, las preocupaciones del personal docente por el logro de aprendizajes presentan una frecuencia menor, al igual que los retos relativos al manejo del tiempo en escenarios en línea.

Las evidencias de los retos según los puntos de vista del personal docente de inicial y primaria que participaron de la encuesta, reflejan los desafíos que han encontrado para desarrollar la enseñanza remota de emergencia. A continuación, se muestran fragmentos de respuestas de docentes de estos niveles educativos (ver Figura 1).

Figura 1: Retos de la enseñanza remota de emergencia, según docentes de Educación Inicial y Primaria $\left(^{*}\right)$

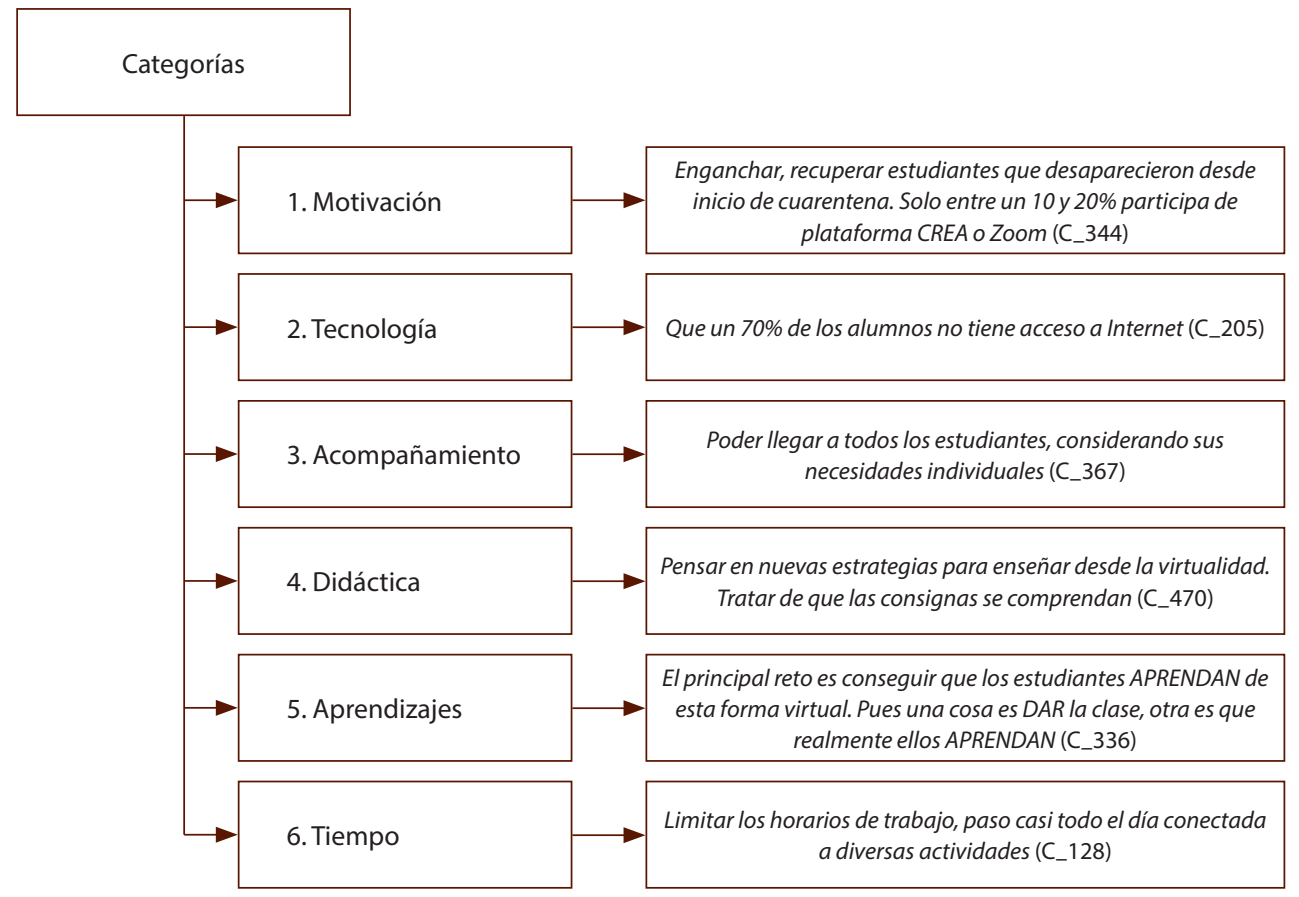

Nota: Elaboración propia. 
https://doi.org/10.15359/ree.26-1.4

https://www.revistas.una.ac.cr/index.php/educare

educare@una.ac.cr

En forma coincidente, el personal docente de educación media visualiza la motivación del estudiantado y la participación en las propuestas como el mayor reto de la enseñanza remota de emergencia. Otro de los desafíos que plantean para esta modalidad de enseñanza es el logro del acompañamiento a la población estudiantil. En el mismo sentido, algunos y algunas docentes señalaron la preocupación por el desarrollo de actividades educativas adaptadas a la enseñanza en línea, las dificultades tecnológicas, los aprendizajes de las personas estudiantes y el tiempo para gestionar el trabajo remoto.

En la Figura 2, las evidencias muestran algunas visiones sobre las categorías mencionadas como retos con mayor frecuencia. Por ejemplo, resulta llamativo que, a pesar de los recursos tecnológicos brindados por el Plan Ceibal, la población estudiantil tenga dificultades para trabajar en la plataforma por no contar con computadora y conexión a Internet. Seguramente por esta dificultad, el personal docente sienta que debe acercar los contenidos en diferentes formatos para lograr el acompañamiento en las trayectorias individuales de los y las estudiantes, y esto les insuma mayores tiempos de trabajo que la modalidad presencial.

Figura 2: Retos de la enseñanza remota de emergencia, según docentes de Educación Media

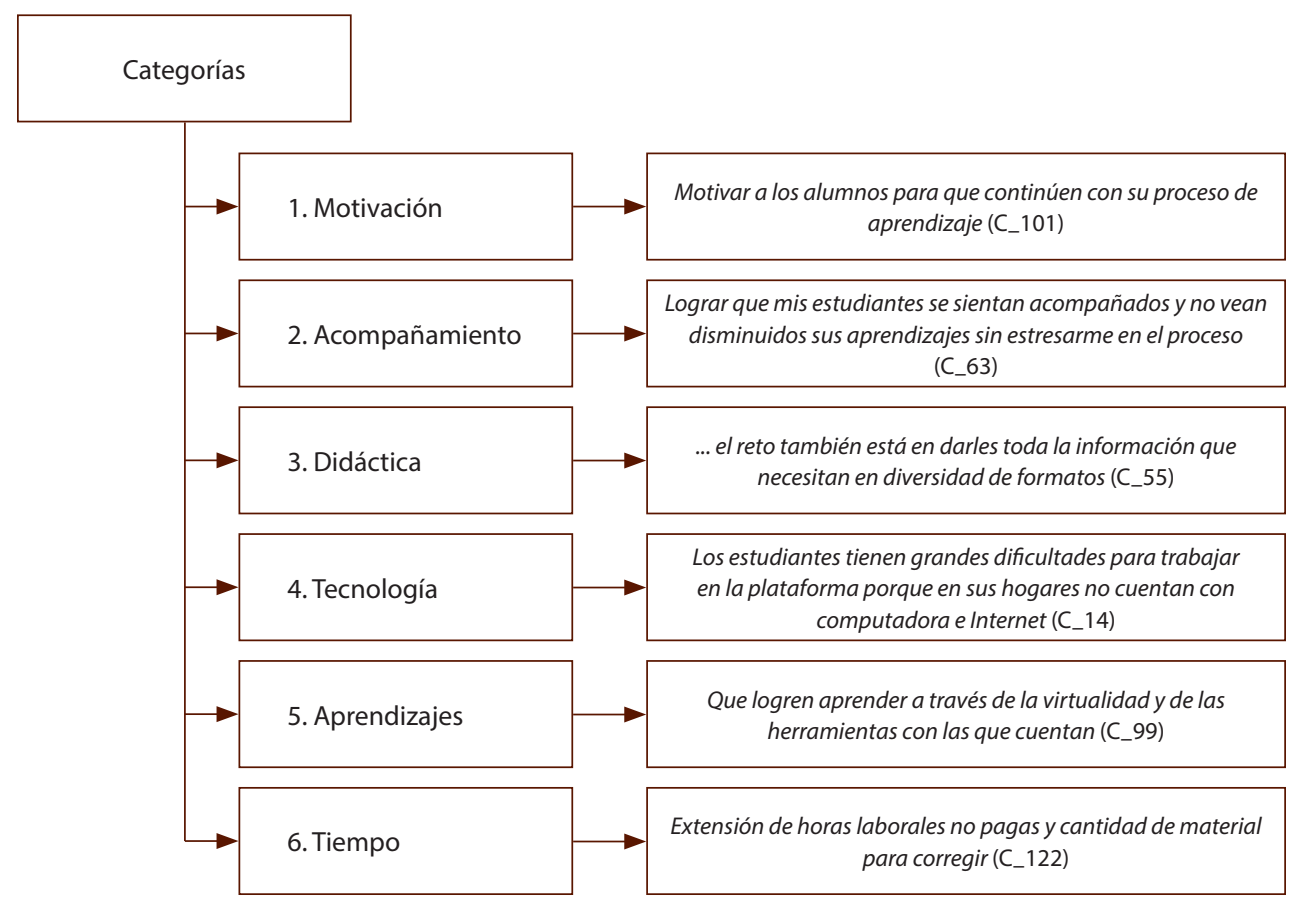

Nota: Elaboración propia. 
https://doi.org/10.15359/ree.26-1.4

Si se tienen en cuenta los retos que identifica el personal docente de educación terciaria, en primer lugar, según el número de frecuencias se encuentran, coincidentemente con el personal docente de otros niveles educativos, los desafíos relativos a la motivación y la participación de los y las estudiantes. Es en este nivel educativo, es donde la didáctica y el logro de los aprendizajes de la población estudiantil tienen mayor relevancia. Los datos confirmar los hallazgos de estudios previos referidos a las dificultades de uso de las tecnologías en la formación de docentes (Marcelo y Vaillant, 2019). Por otra parte, el tiempo necesario para desarrollar las propuestas de enseñanza remota, la disponibilidad de tecnologías y el acompañamiento, son los aspectos que se destacan en cuarto lugar de la categorización por frecuencias de los retos.

La evidencia al respecto de esta categorización muestra que los retos identificados tienen aspectos en común con los demás niveles educativos, en especial la preocupación por la didáctica, el logro de los aprendizajes, y el acompañamiento (ver Figura 3).

Figura 3: Retos de la enseñanza remota de emergencia, según docentes de Educación Terciaria

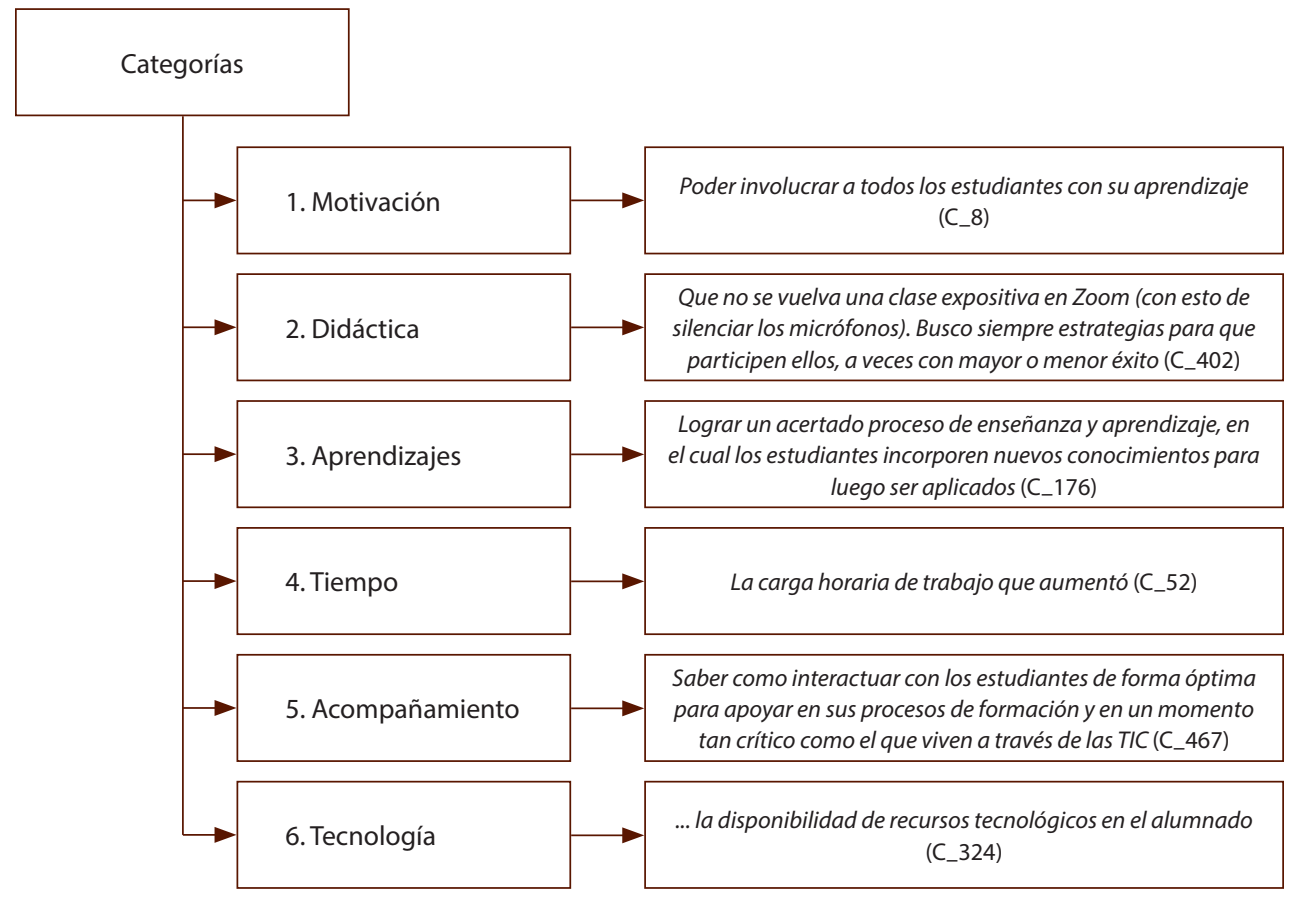

Nota: Elaboración propia.

\section{Discusión y notas finales}

Las evidencias del estudio realizado muestran que incluso en casos de alta conectividad educativa, como el caso uruguayo, el aprendizaje en línea produce tensiones de diversa índole e intensidad. Al igual que en el resto de los sistemas educativos del mundo los resultados 
https://doi.org/10.15359/ree.26-1.4

https://www.revistas.una.ac.cr/index.php/educare

educare@una.ac.cr

evidencian problemas en el ámbito de la salud ocupacional ya que según las percepciones del personal docente encuestado, la enseñanza remota de emergencia implicó, con frecuencia, altos niveles de estrés.

Los docentes encuestados informan acerca de la multiplicidad de estrategias pedagógicas y modalidades de enseñanza implementadas durante la pandemia, pero al mismo tiempo identifican brechas en el acceso y uso de las tecnologías por parte de maestros y profesores. Parecería que aún en países como el Uruguay con altos niveles de acceso a los recursos tecnológicos, no siempre existe un uso pedagógico intensivo de las herramientas digitales a nivel del aula.

En la discusión de los resultados de nuestro estudio es insoslayable mencionar al Plan Ceibal, que ha sido un referente constante en la política educativa nacional implementada desde hace quince años. El andamiaje e infraestructura del Plan permitieron en muy corto plazo, facilitar el acceso de estudiantes y docentes a diversas modalidades de educación en línea. El foco de nuestro estudio no estuvo en el Plan Ceibal, pero resultó atravesado por él pues conlleva particularidades importantes referidas a las políticas digitales de acceso universal uno a uno. Ello hacía suponer que el país iba a implementar modalidades a distancia en muy corto plazo y con éxito para hacer frente a la pandemia. Sin embargo, y de acuerdo con las percepciones del personal docente presentadas en este estudio, aún persisten problemas de baja apropiación y uso educativo de las tecnologías tanto por parte del estudiantado como del personal docente. Este hecho, es especialmente grave si se consideran las brechas digitales en función del contexto y la vulnerabilidad social de las familias (ANEP, 2020a).

El análisis de la información recolectada pone de manifiesto que para el personal docente participante en la encuesta, el pasaje de la educación presencial a la modalidad virtual dificultó el vínculo de la persona docente con el alumnado, así como el acompañamiento y seguimiento del estudiantado con mayores dificultades de aprendizaje, que generalmente pertenecen a familias de menor capital cultural y tecnológico lo que es congruente con los hallazgos de la literatura (Alarcón y Méndez, 2020; Fondo de las Naciones Unidas para la Infancia [UNICEF], 2021).

Otro aspecto por resaltar en los hallazgos refiere al desarrollo de nuevos modelos pedagógicos para la educación a distancia. Las percepciones de los y las docentes son concluyentes respecto a la necesidad de avanzar significativamente en los usos didácticos de las tecnologías digitales. Lo anterior va en línea con las conclusiones de la literatura (Vaillant et. al, 2020) en cuanto a la necesidad de construir nuevos formatos que potencien un uso pedagógico de la tecnología para la mejora de los aprendizajes y el logro de mayores niveles de equidad. 


\section{Referencias}

Administración Nacional de Educación Pública. (2020a). Censo nacional docente 2018. Informe de resultados. https://censodocente2018.anep.edu.uy/censo/documentos/CENSO Nacional Docente LIBRO.pdf

Administración Nacional de Educación Pública. (2020b). Situación educativa en el contexto de la emergencia sanitaria. Encuesta Docente. https://www.anep.edu.uy/15-d/encuesta-paradocentes-aporta-valiosa-informaci-n-en-el-retorno-aulas

Alarcón, A. y Méndez, G. (2020). Informe Uruguay. Seguimiento del retorno a las clases presenciales en centros educativos en Uruguay. Fondo de las Naciones Unidas para la Infancia. UNICEF. https://www.unicef.org/uruguay/informes/seguimiento-del-retorno-las-clasespresenciales-en-centros-educativos-en-uruguay

Alonso, C., Betancor, G., Borba, E., Cuevasanta, D., de Armas, A., Emery, C., González, M., López, R., Lucián, E., Méndez, I., Moreno, L., Núñez, M., Padula, D., Panizza, M. E., Rajchman, A. y Reigosa, V. (2020). Aristas 2018. Informe de resultados de tercero de educación media. INEEd. https://www.ineed.edu.uy/images/Aristas/Publicaciones/Aristas2018/Aristas-2018Informe-de-resultados.pdf

Alonso, C., Cuevasanta, D., Dodino, L., Emery, C., Figueroa, V., García, E., Haretche, C., Mels, C., Méndez, I., Moreno, L., Núñez, M., Padula, D., Panizza, M. E., Picaroni, B., Rajchman, A., Rodríguez, P. y Martín Soca, J. (2018). Aristas 2017. Informe de resultados de tercero y sexto de educación primaria. INEEd. https://aristas2017.ineed.edu.uy/InformeAristas2017.pdf

Bolívar, A., López Yáñez, J. y Murillo, F. J. (2013). Liderazgo en las instituciones educativas: Una revisión de líneas de investigación. Revista Fuentes, (14), 15-60. https://revistascientificas. us.es/index.php/fuentes/article/view/2352/2171

Bühl, V. (2013). Los entornos virtuales de aprendizaje y sus usos en la enseñanza universitaria. Estado de situación y buenas prácticas en las Facultades de Química e Ingeniería de la Universidad de la República [Tesis de maestría]. Universidad de la República. https://www.cse.udelar.edu.uy/

Cabrera Borges, C. A., Rodríguez Zidán, C. E. y Zorrilla Zalgador, J. P. (2018-2019). Integración de dispositivos móviles en la formación inicial y en las prácticas educativas de los estudiantes de profesorado de Uruguay. Revista Latinoamericana de Educación Comparada, 9(14), 123141. https://www.saece.com.ar/relec/numero14.php

Cáceres-Muñoz, J., Jiménez Hernández, A. S. y Martín-Sánchez, M. (2020). Cierre de escuelas y desigualdad socioeducativa en tiempos del COVID-19. Una investigación exploratoria en clave internacional. Revista Internacional de Educación para la Justicia Social, 9(3), 199-221. https://doi.org/10.15366/riejs2020.9.3.011 
https://doi.org/10.15359/ree.26-1.4

https://www.revistas.una.ac.cr/index.php/educare

educare@una.ac.cr

Canal Oficial de la Unidad Académica de Tecnología Educativa del Consejo de Formación en Educación (Uruguay). (6 de agosto de 2020). Relatos docentes en tiempos de pandemia [Video Youtube]. YouTube. https://www.youtube.com/watch?v=b|QIIKq4fp8

Cardozo, S., Retamoso, A. y Biramontes, T. (2020). Situación educativa en el contexto de la emergencia sanitaria. Encuesta docente. - ANEP. https://observatorio.anep.edu.uy/sites/default/files/ arch/Informe\%20Encuesta\%20Docente\%20ANEP\%20-\%2015\%20de\%20julio\%20v2.pdf

Cobo, C. y Montaldo, M. (2018). Plan Ceibal in Uruguay: How do you educate in learning to decode the unknown? IBE; UNESCO. http://repositorio.minedu.gob.pe/handle/20.500.12799/6005

Fondo de las Naciones Unidas para la Infancia. (2021). Informe Argentina. Encuesta de percepción y actitudes de la población. El impacto de la pandemia COVID-19 y las medidas adoptadas por el gobierno sobre la vida cotidiana. Autor.

Hodges, C., Moore, S., Lockee, B., Trust, T. y Bond, A. (2020, March 27). The difference between emergency remote teaching and online learning. EDUCAUSE Review. https://er.educause. edu/articles/2020/3/the-difference-between-emergency-remote-teachingand-onlinelearning

Lamschtein, S. (2018). Tecnologías digitales y aprendizajes visibles. Fundación Ceibal. https:// digital.fundacionceibal.edu.uy/jspui/handle/123456789/289

Lee, J. (2020). Mental health effects of school closures during COVID-19. The Lancet. Child \& Adolescent Health, 4(6), 421. https://doi.org/10.1016/S2352-4642(20)30109-7

Li, Y. (2020). Linking organizational justice to affective commitment: The role of perceived supervisor support in Chinese higher education settings. Asia-Pacific Journal of Teacher Education, 48(3), 237-250. https://doi.org/10.1080/1359866X.2018.1542660

Luengo, F. y Manso, J. (Coords.) (2020). Informe de investigación COVID19. Voces de docentes y familias. Proyecto Atlántida. https://repositorio.uam.es/handle/10486/691408

Lugo, M. T. y Delgado, L. (2020). Hacia una nueva agenda educativa digital en América Latina. CIPPEC. https://www.cippec.org/publicacion/hacia-una-nueva-agenda-educativa-digitalen-america-latina/

Marcelo, C. y Vaillant, D. (2019). Hacia una formación disruptiva de docentes: 10 claves para el cambio. Narcea.

Miles, M. B. y Huberman, A. M. (1994). An expanded sourcebook. Qualitative data analysis (2. ed.). Sage.

Pedró, F. (2020). COVID-19 y educación superior en América Latina y el Caribe: Efectos, impactos y recomendaciones políticas. Fundación Carolina. https://doi.org/10.33960/AC 36.2020 
https://doi.org/10.15359/ree.26-1.4

Perera, M. y Aboal, D. (2017). Evaluación del impacto de la plataforma adaptativa de matemática en los resultados de los aprendizajes. CINVE.

Plan Ceibal. (2020). Ceibal en cifras. https://www.ceibal.edu.uy/es/articulo/ceibal-en-cifras

Plan Ceibal. (s.f.). Qué es Plan Ceibal. https://www.ceibal.edu.uy/es/institucional

Rama, C. (2020). Ejes problemáticos de la equidad de la educación superior en Uruguay. Debate Universitario, 8(16), 27-37. http://portalreviscien.uai.edu.ar/ojs/index.php/debateuniversitario/article/view/236

Reimers, F. M. y Schleicher, A. (2020). A framework to guide an education response to the COVID-19 pandemic of 2020. OECD. https://www.aforges.org/wp-content/uploads/2020/04/ framework.pdf

Rodríguez Zidán, E., Marcelo, C., Bernasconi, G., Yot, C., Téliz, F. y Umpiérrez, S. (2017). Educadores en la era digital: Aprender a enseñar con tecnologías en la formación inicial de profesores de educación media en Uruguay. ANII; Fundación Ceibal. https://digital.fundacionceibal.edu. uy/jspui/handle/123456789/222?mode=full

Sosa, F. y Ambrosi, N. (2021). El rol docente en contexto de pandemia por covid-19. Sistematización de prácticas docentes en la educación pública uruguaya. UNICEF Uruguay; Plan Ceibal; Administración Nacional de Educación Pública. https://www.unicef.org/uruguay/media/5571/ file/El\%20rol\%20docente\%20en\%20contexto\%20de\%20pandemia\%20por\%20Covid-19.pdf

United Nations Educational, Scientific and Cultural Organization. (2020). COVID-19 impact on education. https://en.unesco.org/COVID19/educationresponse

Vaillant, D. (2012). El Plan Ceibal y las prácticas de los docentes de matemática en primer año de liceo (Informe final). ANII; Fundación Ceibal. https://digital.fundacionceibal.edu.uy/jspui/ handle/123456789/139

Vaillant, D., Rodríguez Zidán, E. y Bentancor, G. (2020). La incorporación de herramientas y plataformas digitales en la enseñanza de la matemática. En D. Vaillant (Dirección) y Y. Ferreira (Edición), Educar y aprender en la era digital: Una mirada desde la investigación (pp. 177-195). Universidad ORT Uruguay. https://doi.org/10.1590/s0104-40362020002802241 